\title{
Effect of Nitric Oxide on Endothelial Differentiation of Mesenchymal Stem Cells
}

\author{
Nan Wang \\ Tianjin University of Science and Technology \\ Key Laboratory of Industrial Microbiology \\ Tianjin, China \\ Wn929@tust.edu.cn \\ Li-Nan Zhang \\ Tianjin University of Science and Technology \\ Key Laboratory of Industrial Microbiology \\ Tianjin, China \\ 345497531@qq.com \\ Rui Zhang \\ Tianjin University of Science and Technology \\ Key Laboratory of Industrial Microbiology \\ Tianjin, China \\ 2456845111@163.com
}

\author{
Na Huang \\ Tianjin University of Science and Technology \\ Key Laboratory of Industrial Microbiology \\ Tianjin, China \\ 541810464@qq.com \\ Zhi-Xia Guo \\ Tianjin University of Science and Technology \\ Key Laboratory of Industrial Microbiology \\ Tianjin, China \\ 1083719547@qq.com \\ Tong-Cun Zhang \\ Tianjin University of Science and Technology \\ Key Laboratory of Industrial Microbiology \\ Tianjin, China \\ tony@tust.edu
}

\begin{abstract}
Nitric oxide (NO), as a signaling molecule in vivo, plays an important regulatory role in a variety of physiological and pathological conditions. Mesenchymal stem cells (MSCs) are pluripotent progenitors that can differentiate into a variety of cell types. Vascular endothelial growth factor (VEGF) induces MSC differentiation towards endothelial cells (ECs). Although nitric oxide signaling promotes differentiation and maturation of endothelial progenitor cells, its role in endothelial differentiation of mesenchymal stem cells (MSCs) remains controversial. Frist, cellular proliferation was tested in MSCs treated with/without NO donors S-Nitrosoglutathione (GSNO) or Sodium Nitroprusside (SNP) by MTT assay. Next, after MSCs were cultured with different concentrations of NO donors in the presence of VEGF for 7 days, the cellular morphology was observed under phase microscope and the level of endothelial cell markers fms-like tyrosine kinase (Flt1) was detected by RT-PCR and immunocytochemistry. The results determined that $\mathrm{NO}$ donors $(1-200 \mu \mathrm{mol} / \mathrm{mL})$ had no effect on the survival of MSCs. VEGF-treated MSCs exhibited an endothelial-like phenotype, while NO donorstreated cells still have a spindle-shaped phenotype like MSCs. VEGF treatment increased Flt-1 expression, whereas NO donors inhibited VEGF-induced Flt-1 expression. Our Results suggested that NO could inhibit endothelial differentiation of mesenchymal stem cells.
\end{abstract}

Key words: mesenchymal stem cells; Nitric oxide; differentiation; endothelial cells; RT-PCR

\section{INTRODUCTION}

Mesenchymal stem cells (MSCs), a population of pluripotent progenitors, can differentiate into osteoblasts, chondrocytes and adipocytes. Because of the multipotent differentiation potential and immunosuppressive properties, MSCs have been widely studied and used for regenerative medicine and tissue engineering ${ }^{[1-3]}$. Vascular endothelial growth factor (VEGF) has been shown to stimulate endothelial cells differentiation. Bone marrow-derived MSCs are known to be capable of differentiating into endothelial cells in present of VEGF and $2 \%$ fetal bovine serum (FBS) ${ }^{[4-5]}$.

Nitric oxide (NO), as a gas signal molecule in the body, plays an important role for cellular function, in a variety of physiological and pathological conditions. NO signaling can promote differentiation and maturation of endothelial progenitor cells. However, its role in the endothelial differentiation of MSCs remains controversial. Therefore, the objective of this research is to investigate the role of $\mathrm{NO}$ in endothelial cell differentiation of mesenchymal stem cells ${ }^{[6]}$.

\section{MATERIALS AND METHODS}

\section{A. Cell culture}

Rat bone marrow-derived MSCs (rBM-MSCs) were obtained from tibias and femurs of the male of SpragueDawley (SD) rats (weight $80 \mathrm{~g}$ ) as described previously ${ }^{[2]}$. rBM-MSCs were isolated by Percoll $(1.073 \mathrm{~g} / \mathrm{mL})$ density gradient centrifugation. All cells were maintained at $37{ }^{\circ} \mathrm{C}$ with $5 \% \mathrm{CO}_{2}$ and were cultured basal DMEM-LG medium with 20\% (vol/vol) FBS (Atlanta Biologicals), 1\% (vol/vol) penicillin and streptomycin. Media were replaced every two days. 


\section{B. Cell treatment}

Cells were seeded in 24-well by $5 \times 10^{4}$ cells per well. After cultivated overnight, cells were cultured with basal DMEM-LG medium and $2 \%$ FBS for $24 \mathrm{~h}$ and then incubated with NO donors GSNO $(1-200 \mu \mathrm{mol} / \mathrm{mL})$ or SNP $(1-200 \mu \mathrm{mol} / \mathrm{mL})$ for another $24 \mathrm{~h}$.

\section{MTT assay}

Cell proliferation was quantified using MTT assay. Cells were collected and seeded in 96-well plates by 5000 cells per well. MTT reagent was added $20 \mu \mathrm{L}$ and incubated at $37{ }^{\circ} \mathrm{C}, 5 \% \mathrm{CO}_{2}$ for $4 \mathrm{~h}$. After the incubation, the solution was decanted and added $100 \mu \mathrm{L}$ dimethyl sulfoxide (DMSO) to dissolve the purple formazan crystals, and the culture plate was shaken at low speed for $10 \mathrm{~min}$ until the crystals dissolved completely. The absorbance of the resulting solution was measured at $570 \mathrm{~nm}$ with a microplate reader.

\section{Cell differentiation induction}

rBM-MSCs were cultured in basal DMEM-LG medium with 50ng/mL VEGF (Peprotech), 2\% FBS and $1 \%(\mathrm{vol} / \mathrm{vol})$ penicillin and streptomycin, and grown under at $37{ }^{\circ} \mathrm{C}$ with $5 \% \mathrm{CO}_{2}$ for 7 days. Media were changed every two days. These cells were observed under phase microscope. For NO donor treatment, MSCs were cultured with $100 \mu \mathrm{mol} / \mathrm{mL}$ SNP (Sigma) in the presence of VEGF for 7 days.

\section{E. Reverse-transcription polymerase chain reaction (RT-PCR)}

Total RNA was isolated from cells using Trizol reagent (Invitrogen), and $2 \mu \mathrm{g}$ of the sample were reversetranscribed using M-MLV reverse transcriptase. The thermal cycle profile was as follows: denaturation for $30 \mathrm{~s}$ at $95{ }^{\circ} \mathrm{C}$, annealing for $45 \mathrm{~s}$ at $50-54{ }^{\circ} \mathrm{C}$ depending on the primers used, and extension for $30 \mathrm{~s}$ at $72{ }^{\circ} \mathrm{C}$. PCR products were visualized on $2 \%$ agarose gels stained with ethidium bromide under UV transillumination. Glyceraldehyde-3-phosphate dehydrogenase (GAPDH) was used as a housekeeper. The primers are as follows: Flt-1:forward-CGGAGAAATCTGCTCGCTAT, reverseCTTGGAAGGGACGACACG; GAPDH:forwardATTCAACGGCACAGTCAAGG-3, reverseGCAGAAGGGGCGGAGATGA

\section{F. Immunocytochemistry assay}

The cells were fixed with $4 \%$ paraformaldehyde for 15 min, and blocked with normal goat serum for $20 \mathrm{~min}$ at room temperature (RT). After incubation with rabbit antiFlt-1(Abcam) overnight at $4^{\circ} \mathrm{C}$, cells were incubated with appropriate secondary antibodies (FITC-conjugated goat anti-rabbit $\mathrm{IgG}$, Santa Cruz) for $1 \mathrm{~h}$ at $37^{\circ} \mathrm{C}$. Then, the samples were washed with PBS and observed under laser scanning confocal microscope (OLYMPUS). DAPI stain (blue) highlights the total nuclei.

\section{G. Statistical analysis}

Data were presented as means \pm SD. The significant difference was examined using the Student's t test. The minimal level of significance was $\mathrm{P}<0.05$.

\section{RESULT}

\section{A. The phenotype of Mesenchymal stem cells.}

All cells were cultured and observed by inverted phase contrast microscope. Cells were spindle cell-based, showing radial colony arrangement after continuous 3 passages.

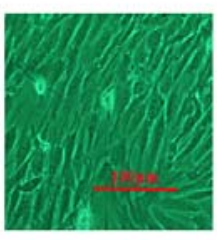

P1

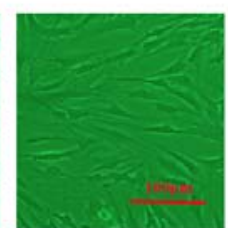

P2

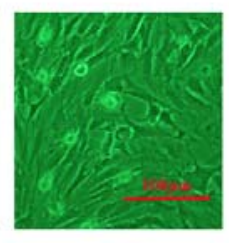

P3
Figure1. Mesenchymal stem cells were observed inverted phase contrast microscope.

\section{B. NO stimulation does not affect the proliferation of MSCs.}

In order to study whether NO can affect the proliferation of bone marrow mesenchymal stem cells, NO donors GSNO $(1-200 \mu \mathrm{mol} / \mathrm{mL})$ or SNP $(1-200 \mu \mathrm{mol} / \mathrm{mL})$ were added in rBM-MSCs for 24h. As shown in Fig.2, We found that NO donors unaffected the proliferation of MSCs.
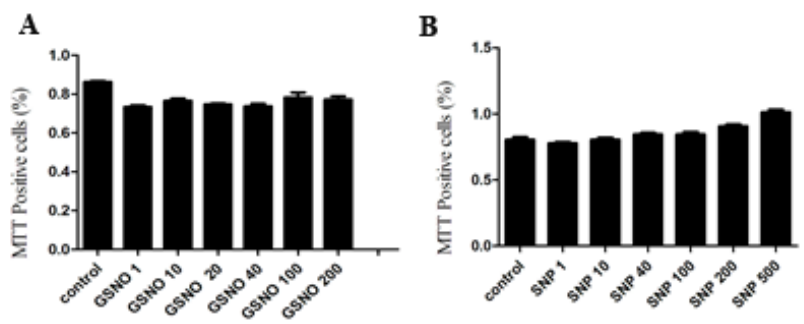

Figure2. NO stimulation does not affect the proliferation of mesenchymal stem cells. A. MTT assay showed that proliferation of MSCs after NO donors GSNO $(1-200 \mu \mathrm{mol} / \mathrm{mL})$ were treated for $24 \mathrm{~h}$. B MTT assay respectively showed that proliferation of MSCs after NO donors SNP $(1-200 \mu \mathrm{mol} / \mathrm{mL})$ were treated for $24 \mathrm{~h}$.

\section{NO inhibits mesenchymal stem cell differentiation.}

In order to study whether NO can influence the differentiation of mesenchymal stem cells, MSCs were treated with $100 \mu \mathrm{mol} / \mathrm{mL} \mathrm{SNP}$ in the presence of $50 \mathrm{ng} / \mathrm{mL}$ VEGF for 7 days and were observed by inverted phase contrast microscope. VEGF-treated MSCs exhibited an endothelial-like phenotype, while NO donors-treated cells, together with VEGF, still have a spindle-shaped phenotype like MSCs (Fig.3). 


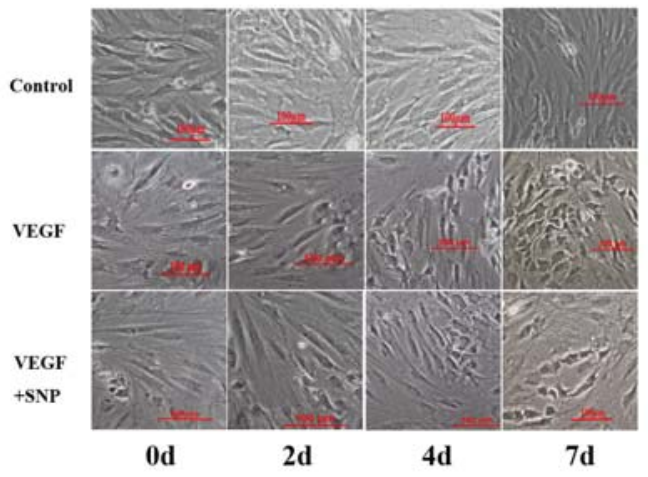

Figure3. NO inhibited mesenchymal stem cell differentiation. Mesenchymal stem cells were observed inverted phase contrast microscope after treatment with $100 \mu \mathrm{mol} / \mathrm{L}$ NO donors SNP and $50 \mathrm{ng} / \mathrm{mL}$ VEGF at 2, 4 and $7 \mathrm{~d}$.

\section{NO stimulation decreased the expression of Flt-1.}

In addition, RT-PCR was performed to detect expression of differentiation marker Flt-1. The mRNA levels of Flt-1 were decreased in a dose-dependent manner (Fig.4A and B).

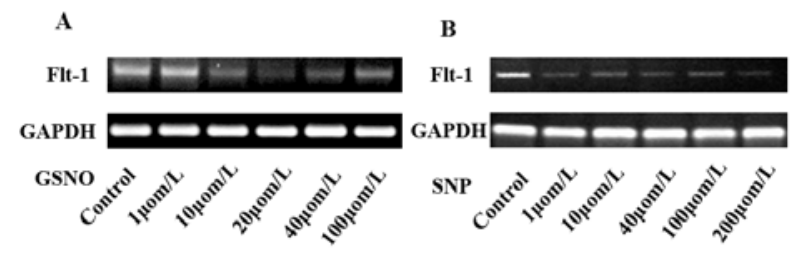

Figure4. NO induced the decreased levels of Flt-1. A. The mRNA levels of the differentiation markers Flt-1 in MSCs treated with GSNO $(1-100 \mu \mathrm{mol} / \mathrm{mL})$ or SNP $(1-200 \mu \mathrm{mol} / \mathrm{mL})$ for $24 \mathrm{~h}$ in the presence/absence of VEGF-induced 7 days.

Next, immunocytochemistry assay was performed. According to the overall fluorescence intensity of specific staining for Flt-1, VEGF-treated differentiated cells have positive staining for Flt-1, while NO-treated cells, together with VEGF, have low Flt-1 expression (Fig.5). These results showed that $\mathrm{NO}$ could inhibit endothelial differentiation of MSCs compared with VEGF alone treated.
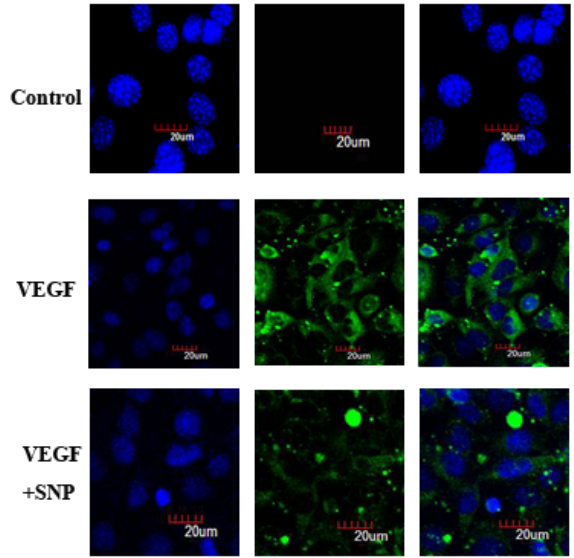

Figure5. Immunostaining and confocal microscopy were used to determine expression of Flt-1.

\section{CONCLUSION}

VEGF has important effects in the endothelial system during development and endothelial differentiation. MSCs do not express VEGF receptors (Flt-1). Previous studies demonstrated that after VEGF treatment, MSCs from rat bone marrow exhibited Flt-1 positive expression and differentiated into endothelial cells [7-9]. A recent publication from Gomes et al. demonstrate that VEGF-A signaling is a key in MSC-mediated vasculogenesis and NO downregulates VEGF-A signaling to reduce tube formation by MSCs, suggesting an opposite role of NO in vascular formation from $\mathrm{MSCs}^{[10]}$. Our studies also showed that NO had inhibitory influence on MSCs differentiation. NO can selectively modify protein cysteine residues to form S-nitrosocysteine. This post-translational modification, S-nitrosylation, impacts protein function, stability, and location. Therefore, these studies will provide a theoretical basis for exploring the process of VEGFinduced bone marrow mesenchymal stem cells differentiation into endothelial cells.

\section{ACKNOWLEDGMENTS}

This work was supported by the National Natural Scien ce Foundation of China (31171303, 31171297, 31270837) and the Program for Changjiang Scholars and Innovative R esearch Team in University of the Ministry of Education of China (IRT1166).

\section{REFERENCES}

[1] Oswald J, Boxberger S, Jorgensen B, et al. Mesenchymal stem cells can be differentiated into endothelial cells in vitro. Stem Cells 2004;22:377-84.

[2] Wang N, Zhang R, Wang SJ, et al. Vascular endothelial growth factor stimulates endothelial differentiation from mesenchymal stem cells via Rho/myocardin-related transcription factor--a signaling pathway. Int J Biochem Cell Biol. 2013 Jul;45(7):144756.

[3] Xu Y, Liu L, Zhang L, et al. Efficient commitment to functional CD34+ progenitor cells from human bone marrow mesenchymal stem-cell-derived induced pluripotent stem cells. PLoS ONE 2012; 7:e34321.

[4] Beckermann BM, Kallifatidis G, Groth A, et al. VEGF expression by mesenchymal stem cells contributes to angiogenesis in pancreatic carcinoma. British Journal of Cancer 2008;99:622-31. 
[5] Wang M, Su Y, Wang F, et al. Induced endothelial differentiation of cells from a murine embryonic mesenchymal cell line $\mathrm{C} 3 \mathrm{H} / 10 \mathrm{~T} 1 / 2$ by angiogenic factors in vitro. Differentiation 2010;79:21-30.

[6] Gomes SA1, Rangel EB, Premer C, et al. S-nitrosoglutathione reductase (GSNOR) enhances vasculogenesis by mesenchymal stem cells. Proc Natl Acad Sci U S A. 2013 Feb 19;110(8):2834-9.

[7] Au P, Tam J, Fukumura D, et al. Bone marrow-derived mesenchymal stem cells facilitate engineering of long-lasting functional vasculature. Blood 2008;111:4551-8.

[8] Ball SG, Shuttleworth CA, Kielty CM. Platelet-derived growth factor receptor-alpha is a key determinant of smooth muscle alpha- actin filaments in bone marrow-derived mesenchymal stem cells. International Journal of Biochemistry and Cell Biology 2007;39:379-91.

[9] Bai K, Huang Y, Jia X, et al. Endothelium oriented differentiation of bone marrow mesenchymal stem cells under chemical and mechanical stimulations. Journal of Biomechanics 2010;43:117681.

[10] Gomes SA, Rangel EB, Premer C, et al. S-nitrosoglutathione reductase (GSNOR) enhances vasculogenesis by mesenchyma stem cells. Proceedings of the National Academy of Sciences of the United States of America 2013;110:2834-9. 